\title{
Analysis of Step Responses in Nonlinear Dynamic Systems Consisting of Antagonistic Involvement of Pneumatic Artificial Muscles
}

\author{
Miroslav Rimár, ${ }^{1}$ Peter Šmeringai, ${ }^{1}$ Marcel Fedak, ${ }^{1}$ Michal Hatala, ${ }^{2}$ and Andrii Kulikov ${ }^{1}$ \\ ${ }^{1}$ Department of Process Engineering, Faculty of Manufacturing Technologies, Technical University of Kosice with a Seat in Prešov, \\ Sturova 31, 08001 Prešov, Slovakia \\ ${ }^{2}$ Department of Manufacturing Technologies, Faculty of Manufacturing Technologies, \\ Technical University of Kosice with a Seat in Prešov, Sturova 31, 08001 Prešov, Slovakia
}

Correspondence should be addressed to Peter Šmeringai; peter.smeringai@tuke.sk

Received 22 October 2016; Revised 2 January 2017; Accepted 10 January 2017; Published 22 February 2017

Academic Editor: Giovanni Berselli

Copyright (C) 2017 Miroslav Rimár et al. This is an open access article distributed under the Creative Commons Attribution License, which permits unrestricted use, distribution, and reproduction in any medium, provided the original work is properly cited.

\begin{abstract}
The paper describes a set of experimental measurements carried out on the experimental equipment with a drive based on pneumatic artificial muscles. Based on the analysis of the PAMS control systems issue in relation to the issue of a position control, a control algorithm has been designed and verified. The requirements of the control systems do not arise only from the condition of the desired positioning point rapid achievement, but also from the subsequent dynamics and accuracy repeatability. This algorithm enables an efficient way of stabilization of the actuator position in various dynamic conditions during the operation. It allows eliminating undesirable vibrations oscillating around the point of the required position and dampening them appropriately. The article describes a set of performed verification experimental measurements confirming the applicability in relation to the system that controls the position of the actuator utilizing the described algorithm. The algorithm application enables a positive influencing and optimization of the actuator positioning accuracy and a full-valued automation of its operation.
\end{abstract}

\section{Introduction}

Research and development in manufacturing technology have been constantly motivated by new challenges in manufacturing companies and firms focused on production of manufacturing machines and manipulators. The effort to keep a strong competitive environment leads manufacturers of manufacturing technology to searching for new solutions to structural nodes in manufacturing technology. It also leads to introduction of modern technology to manufacturing. For instance, the requirements of modern manufacturing devices which are able to perform their functions in aggressive environment are currently very demanded. It also refers to the requirements for an adapted technical device, for example, a device with a modified drive which allows its functioning in the environment where standard types of drives cannot be used due to their negative effect on the work environment.
In terms of the need of technical equipment adjustments, there may occur multiple factors affecting the choice of an equipment suitable replacement; for example, the size of a device and its output with a given size (weight) are important. In this regard, it is possible to consider a more efficient energy utilization in a manufacturing system. Each of the nonconventional drives (electric, pneumatic, or hydraulic) is characterized by its own deficiencies. Considering the drives options, the power-to-weight ratio is the main characteristics thanks to which manipulation devices with power units comprising pneumatic artificial muscles start to be used in production facilities [1-4].

Artificial pneumatic muscles have also started to be applied to medicine [5]. The fundamental principle of a pneumatic artificial muscle (PAM) functioning allows evocating a tensile force in one direction. Its use for the movement in both directions requires its use with the involvement of the 
element for a force bond acting in the opposite direction to a PAM force acting. This may concern the PAM involvement in a binding interaction with a pulley system and an onerous element, elastic element (e.g., a spring), or another artificial muscle.

The paper deals with the utilization of the involvement of two pneumatic artificial muscles in an antagonistic connection [6]. Their wider use is limited due to their significant nonlinearity in cases of higher positioning requirements [7].

In present conditions, when a considerable part of manufacturing facilities is oriented towards automated operation, the method of control of manufacturing devices with drives based on pneumatic artificial muscles has to be considered [8-12].

Nowadays, the term "pneumatic artificial muscle" (PAM) starts to be used more often in connection with propulsion systems. Due to its characteristics, it is used not only in simple applications. Thanks to a sufficiently advanced control computing it becomes a part of even more complex mechanisms or actuators. Due to its characteristics, it allows such applications where, under specified conditions, electric and hydraulic actuators fail. The PAM is characterized by a disposition of a functionality similar to a human muscle. These properties of the PAM are a prerequisite for its use in the areas, such as manufacturing and transportation industries or medicine $[8,9]$.

Regarding the range of utilization, the McKibben type of the artificial muscle is currently most widely used and studied. The McKibben artificial muscle has a number of modifications, such as braided pneumatic actuator, pneumatic muscle-fluidic muscle (FESTO), and rubbertuator. These are modifications of the McKibben artificial muscle that have a significantly high power-to-weight ratio with a sufficient stability of flexibility. Their disadvantage is their nonlinearity and therefore insufficiently solved problems of a positioning accuracy that is needed for a wider use of the PAM in manufacturing industry $[8,9,13-15]$.

\section{Characteristics of Pneumatic Artificial Muscles}

The functional principle of artificial muscles is generally defined as a conversion of pneumatic energy into mechanical energy $[9,15]$. As mentioned in the introduction the artificial muscle is a traction drive whose characteristics approach to those of a biological muscle. It consists of a contractile element, a mounting equipment (to mount the PAM in the stable position), and connecting devices (to connect the pressurized working medium).

With fluid artificial muscles, the maximum tractive force is at the beginning of a contraction. During the lift (a gradual change in length), it quasi-linearly decreases. The term "muscle contraction" refers to shortening of its length. It is expressed in the form of a percentage ratio to a nominal length of an unloaded artificial muscle membrane. From the foregoing it follows that the durability of the artificial muscle is largely dependent upon a contraction and operating pressure as well as temperature $[15,16]$.
Functionally, the operation of the artificial muscle is based on a performance of the operating medium which acts on the inner wall of the artificial muscle (membrane), thus increasing its volume. Increasing volume causes a change in the inclination of the artificial muscle braid which results in the braid shortening. The artificial muscle develops a tensile force by its shortening. Based on the above described factors it follows that artificial muscle reduces its length and increases its volume with the increasing pressure of a working medium $[1,9,13,17]$.

Work carried out by the artificial muscle can be divided into the so-called input work $W_{\text {in }}$ and output work $W_{\text {out }}$ $[1,9,18]$. The input work $W_{\text {in }}$ is carried out by a working medium pushing on the inner surface of the artificial muscle membrane [1]. The input work, acting on the wall of the membrane, is defined as $[1,9]$

$$
d W_{\text {in }}=\int_{S_{i}}\left(P-P_{0}\right) d l_{i} \cdot d s_{i}=P^{\prime} \cdot d V
$$

where $P / P_{0}$ is the absolute pressure of the working fluid inside the muscle/ambient gas, $P^{\prime}$ is the relative pressure (deviation of $P$ and $\left.P_{0}\right), s_{i}$ is the inner surface of the PAM, $d s_{i}$ is the difference in surface, $d l_{i}$ is the displacement of the inner surface, and $d V$ is the total volume change $[1,9]$.

The result of the muscle shortening is the output work $W_{\text {out }}[1,9]:$

$$
d W_{\text {out }}=-F \cdot d l
$$

where $F$ is the tensile force of the PAM acting in the axial direction of the muscle and $d l$ is the axial displacement of the muscle $[1,9]$.

Assuming a simplified system, if its losses are neglected, the output work is equal to the input work $[1,9]$ :

$$
d W_{\text {out }}=d W_{\text {in }}
$$

Substituting the right sides of (1) and (2) into (3) we obtain $[1,9,11]$

$$
-F \cdot d l=P^{\prime} \cdot d V
$$

After the adjustment,

$$
F=-P^{\prime} \cdot \frac{d V}{d l} .
$$

Provided that the shape of the active part of the artificial muscle is simplification into the ideal shape of a cylinder (Figure 1), where the length of the muscle $l$ is a representation of the ideal height of the cylinder, $\theta$ is the angle of the inclination of the muscle braid fibers from the ideal axis of the cylinder, $n$ is the number of encircles of network structure fibers around the ideal cylinder of the muscle, $D$ is a diameter of the cylinder, and $b$ is a total length of the fiber, we can estimate the volume change according to the change in length of the cylinder $(d V / d l)$.

The length $l$ and the diameter $D$ of the cylinder can be expressed as the function of the angle of the cylinder 


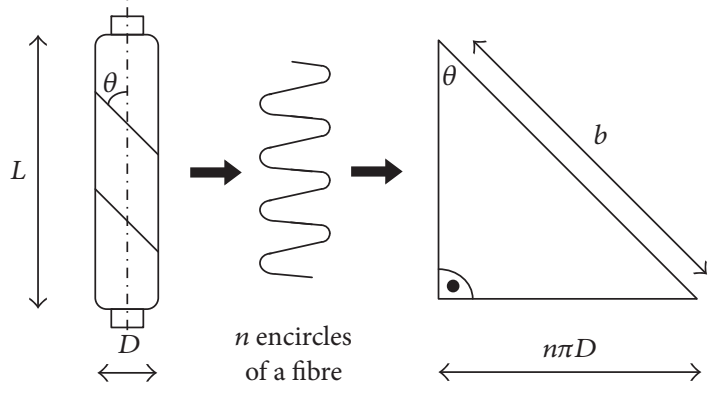

FIGURE 1: Functional principle of the artificial muscle braid [1].

inclination $\theta$ with constant quantities $n$ and $b$ indicating the characteristics of a particular cylinder $[1,9]$ :

$$
\begin{aligned}
l & =b \cdot \cos \theta \\
D & =\frac{b \cdot \sin \theta}{n \cdot \pi} .
\end{aligned}
$$

Subsequently, it is possible to determine the volume of the ideal cylinder as $[1,9]$

$$
V=\frac{1}{4} \cdot \pi \cdot D^{2} \cdot l=\frac{b^{3}}{4 \pi \cdot n^{2}} \cdot \sin ^{2} \theta \cdot \cos \theta .
$$

Equation (5): after these modifications, it is possible to derive the resulting dependence of the muscle tensile force $F$ on the relative pressure $P^{\prime}$ and the inclination of fibers from the cylinder axis $\theta[1,9]$ :

$$
F=-P^{\prime} \cdot \frac{d V}{d l}=-P^{\prime} \frac{d V / d \theta}{d l / d \theta}=\frac{P^{\prime} \cdot b^{2}\left(3 \cos ^{2} \theta-1\right)}{4 \pi \cdot n^{2}} .
$$

Substituting from the right side of (6), it is alternatively possible to express the force as a function of $P^{\prime}$ and $l[1,9]$ :

$$
F=\frac{P^{\prime} \cdot\left(3 l^{2}-b^{2}\right)}{4 \cdot \pi \cdot n^{2}} .
$$

Of the previously described relations it follows that the dependence of a tensile force and pressure of the working fluid inside the ideal cylinder (representing a simplified artificial muscle) is liable to a direct proportion and a monotone function of fibers inclination from the cylinder axis $[1,9]$.

From the foregoing and in accordance with [15] it ensues that the basic possible uses of artificial muscles are

(i) single-acting drive,

(ii) pneumatic spring.

The PAM cannot be used as an air spring without a sufficient operating pressure [15].

\section{Materials and Methods}

3.1. Preparation of Experimental Measurements. The aim of the experiment realization was to verify the algorithm modularization for controlling the position of the PAMs enabling an effective way of the actuator position stabilization in various dynamic conditions during the operation in relation to the positioning accuracy. In experiments, during the changes in position, there was monitored the influence of a static load placed on the arm of the experimental assembly on the dynamics of the actuator arm stabilization process utilizing the power based on the pneumatic artificial muscles in antagonistic involvement. There was also observed the effect of the proposed position control algorithm on the inaccuracies elimination. The effort was to achieve a stable position.

In order to measure the position of the arm, the repeated sequences were performed in which the characteristics to achieve the desired point and the actual dynamics of the process were followed, that is, the rate of acceleration in stabilizing and its impact on the process of stabilization. Owing to the nature of the system and its behaviour in a direct relation to the stiffness, each of the defined characteristic points was monitored. Increment of the change in a position was set from a zero starting position up to the maximum value. Based on the research experiments, according to [9], it is not appropriate to use the PWM in the range below $20 \%$, considering the extreme decrease in durability of the electropneumatic valves. Therefore, the range from 0 to $15 \%$ was indicated as unused in the implementation of the experiment. As the first value, $15^{\circ}$ deflection change was determined, while all following values had increment of $15^{\circ}$.

The methodology of the procedure to achieve the objective of the experimental measurements was guided by the following scheme (Figure 2).

Within the elaboration of the methodology for measuring the transient characteristics of the PAM, the initial requirements of the functionality of the PAMs control system have been set. The control system has been designed to allow a modification to its functionalities on the modular arrangement of algorithms. The method, respectively, procedure for analyzing the state of the system during each iteration was designed. The designed methodology is not based only on the premise of the PAMs state online analysis. It is also based on the need of the analysis of the system after its operation using the data taken during its operation. The measuring of the system state consists of the PAMs measurements of variables commonly measured on drives working based on the pneumatic artificial muscles, such as the pressure in each of the PAM and the position of the support arm. These variables provide information about the state of the system but do not follow the processes taking place inside each PAM and inside the transport routes of the working fluid. Similarly, they do not allow studying the effect of nonlinearity of the PAMs system. Therefore, the question arose about how to follow the dependence of processes taking place inside the PAMs system. At the same time the requirement arose of the smallest possible interaction of sensing devices with the PAMs system in terms of a minimal impact on the functionality and the system load. Therefore, a measurement of a supporting arm acceleration was added. Monitoring of this physical quantity envisaged the possibility of a future description of transitional processes inside the PAMs. There were taken into account the conditions of the influence of pressure of the working fluid and the time of the working fluid acting on the 


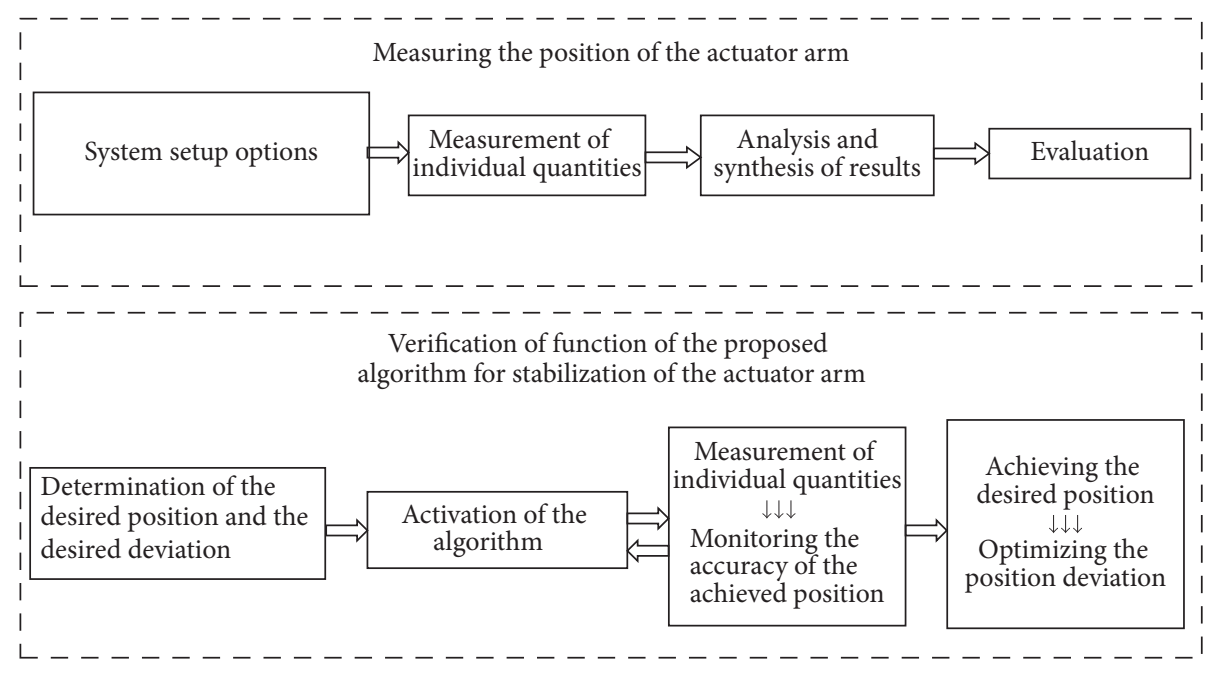

FIGURE 2: Schematic representation of the methodology of the measurement procedures.

acceleration of the loaded system supporting element with the known pressures in each PAM.

For the measurements, there were performed three experiments. In the experiment number 1 , the measurements were carried out under conditions based on the known knowledge. Then, on the basis of the data obtained in the experiment number 1, corrections were performed and verified in experiments numbers 2 and 3.

3.2. Characteristics of the Experimental Device. At the Department of Process Technique, the Faculty of Manufacturing Technologies with a Seat in Prešov, an experimental assembly of an actuator powered by artificial muscles was constructed. The experimental actuator assembly comprises an antagonistic involvement of artificial muscles with a gearing to a rotational movement of a rotary arm, a control and regulation passage, and a pneumatic system supplying a working fluid into the actuator drive. The assembly is completed with the computer control system. The control is programmed in LabVIEW, and its computing hardware base was assembled from the components of the series CompactRIONational Instruments. Figure 3 gives a schematic representation of the experimental device.

The experimental actuator is divided into smaller parts:

(1) Antagonistic involvement of two pneumatic artificial muscles (PAMs).

(2) The system for a drive load.

(3) Pneumatic system.

(4) The system for measurement of dynamic characteristics, monitoring, and control of the experimental device.

(5) Elements of a signal system experimental involvement.

(6) Manual control interface.

The antagonistic involvement of pneumatic artificial muscles is the involvement in which the exerted force is used for a rotary movement of the shaft which is loaded by a supporting rotary arm. In the involvement, two fluidic artificial muscles FESTO MAS-40-1000N-AA-MC-K are used. The PAM inner diameter is $40 \mathrm{~mm}$, a nominal length $1000 \mathrm{~mm}$, operating pressure $0-6$ bar, maximum extension $3 \%$ of its nominal length, and maximum hysteresis $2.5 \%$ of the nominal length. View of the real experimental device is shown in Figure 4.

Compressed air is used as a working medium. A drive load system consists of the pivoting arm which is connected to the drive shaft. The drive shaft is driven via a chain transmission whose chain is connected to each PAM. The size of the load can be controlled via a prismatic connection of the pivot arm and a weight $(5 \mathrm{~kg})$. The pneumatic system ensures the supply of a pressurized fluid by the compressor Güde AL210/8/24E with the air tank. The pressure control inside the pneumatic artificial muscles is realized through proportional electropneumatic valves P/N MX821.104224 which ensure the inflation of a pressurized medium to each PAM as well as its draining. Electropneumatic valves enable the control by a pulse-width modulation (PWM).

The pressure measuring of the operating medium inside the PAM is provided by pressure sensors working on the basis of a ceramic measuring cell. An electric current loop, in which they are connected, works with the electric current in the interval of 4-20 mA. The system for a dynamic characteristics measurement, monitoring, control, and regulation of the experimental device involves computational units that perform tasks of measurement, monitoring, control, and regulation using PC user's connections, manual control unit, and conducting of electrical variables. The essence of control activities across the experimental involvement is based on the control of valves opening and closing using the pulsewidth modulation (PWM). The idea of using the PWM was taken from $[7,8,20]$. In order to ensure measuring, monitoring, and controlling operations online, a real-time computer unit of the CompactRIO, NI cRIO 9024 class was designed. NI cRIO 9024 is connected to the bus NI cRIO 


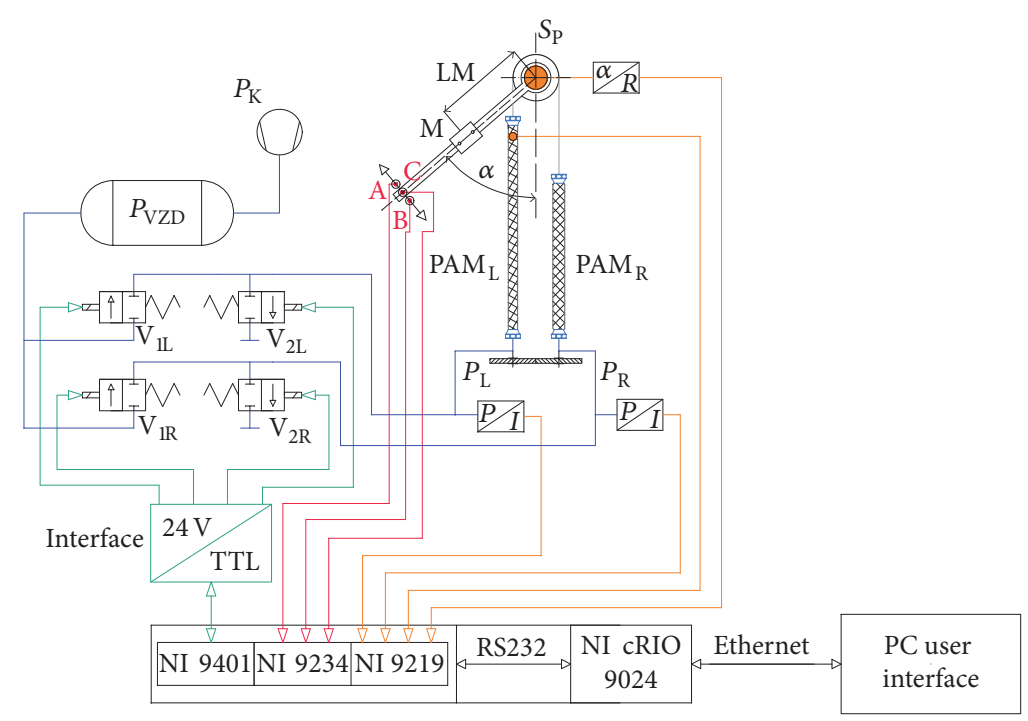

FIGURE 3: Schematic representation of the experimental involvement. $\mathrm{PAM}_{\mathrm{L}}$ : left pneumatic artificial muscle, $\mathrm{PAM}_{\mathrm{R}}$ : right pneumatic artificial muscle, LM: distance of a gravity center of an onerous element $\mathrm{M}$ from the pivoting arm shaft, $P_{\mathrm{R}}$ : right pressure sensor, $P$ : pressure, $S_{\mathrm{P}}$ : potentiometric position sensor, $\alpha$ : rotation angle of the arm from the neutral position, $P_{\mathrm{L}}$ : left pressure sensor, $P_{\mathrm{R}}$ : right pressure sensor, $I$ : electric current, $R$ : electrical resistance, $P_{\mathrm{K}}$ : pressure at the compressor outlet, $\mathrm{V}_{1 \mathrm{~L}} / \mathrm{V}_{1 \mathrm{R}}$ : left/right inflation electropneumatic valve, $\mathrm{V}_{2 \mathrm{~L}} / \mathrm{V}_{2 \mathrm{R}}$ : left/right draining electropneumatic valve, $P_{\mathrm{VZD}}$ : pressure in the air tank pressure, and A/B/C: acceleration sensors. Orange colour: signals from measuring devices, red colour: signals from vibration sensors, green colour: control signals to control electropneumatic valves, and blue colour: transport routes for pressure medium.

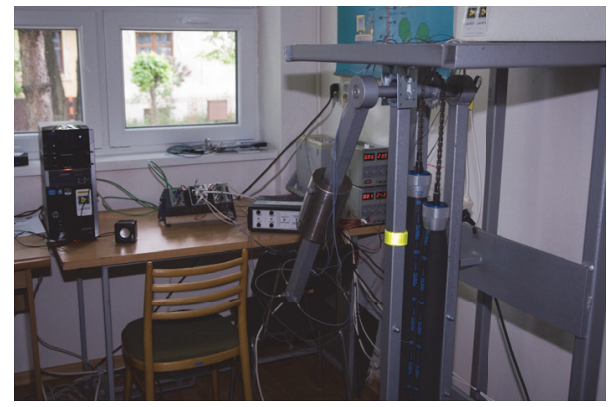

FIgure 4: Experimental device with a drive based on the PAMs.

9114. The bus provides the functionality of its operations with the accuracy of $100 \mathrm{ppm}$ and enables a connection of eight measuring modules. The measured values (pressure at the input into each PAM, arm rotation) are processed as signals by a measuring card NI 9219 with four analog inputs. For electropneumatic valves control, a digital input/output card NI 9041 was used. A direct connection of NI 9041 to the valves was not possible; therefore an independent interface working on the basis of TTL logic $(\mathrm{H} / \mathrm{L} 5 \mathrm{~V})$ was designed. In case that the $5 \mathrm{~V}$ voltage is applied to the circuit, it switches the power supply circuit with $24 \mathrm{~V}$ for the electropneumatic valves. In order to run the service workplace programs, a personal computer is used. The data collection related to the frequency characteristics during the system operation and which referred to the movement of the rotating arm is provided by the measuring card NI 9234 that processes the analog output from the installed vibrometers.
TABLE 1: Acceleration sensors parameters [19].

\begin{tabular}{lcc}
\hline Characteristics & Value & Units \\
\hline Reference sensitivity & 20 & $\mathrm{~m} \cdot \mathrm{s}^{-2} \mathrm{RMS}$ \\
Frequency range & $1-10,000$ & $\mathrm{~Hz}$ \\
Resonant frequency & 32 & $\mathrm{kHz}$ \\
Transversal sensitivity & $<5$ & $\%$ \\
Range of measurement & \pm 4000 & $\mathrm{~m} \cdot \mathrm{s}^{-2}$ peak \\
\hline
\end{tabular}

The elements of the signal system may be divided into elements implemented directly into the experimental device and elements, or measuring devices, the use of which is not required to carry out measurement directly in real time; thus they are used during the device operation sporadically, with a low intensity for creating the indicative overview of the system or as a single application. In the experimental involvement, sensors to measure the acceleration at the end of the supporting rotational arm are installed (Table 1).

For the sensing of the supporting arm rotation size, a potentiometric resistive divider with linear characteristics was used. Its working range is $0-10 \mathrm{k} \Omega$ with a maximum rotation of $270 \pm 5^{\circ}$. To measure the ambient temperature, a thermal hygrometer TESTO $605-\mathrm{H} 1$ was used. The electricity supply for the equipment of the experimental device, which has special requirements of electrical current and voltage, is provided by regulated laboratory power supplies DC Power Supply HY3005. The control system allows performing control tasks only by means of signals for electropneumatic valves opening and closing. For the valves manual control, a separate device (interface for manual control) was built, 


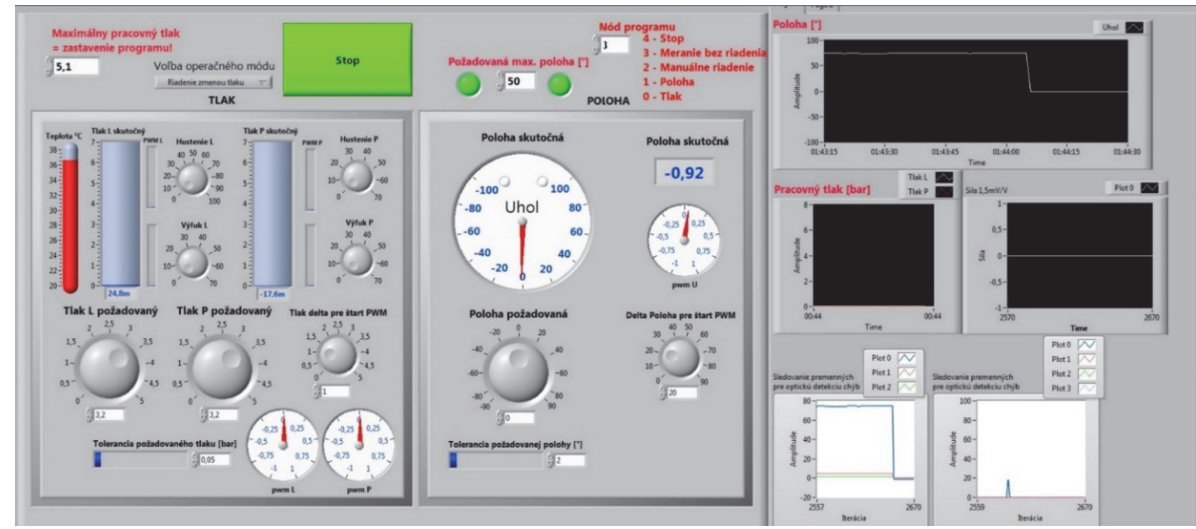

FIgURE 5: Taskbar of the designed user interface.

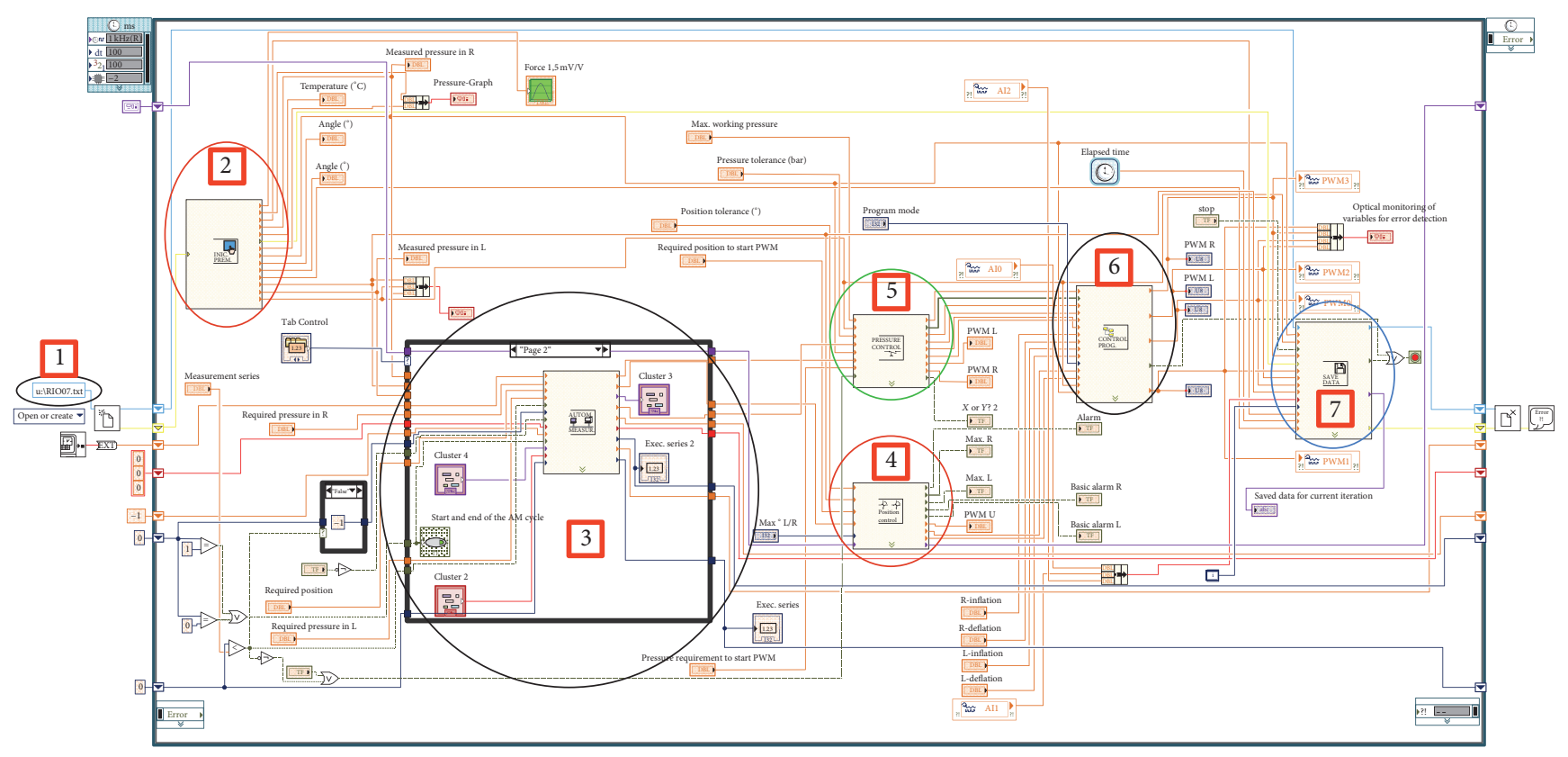

FIGURE 6: Block diagram of the main algorithm.

which is independent of the control system. In case that the control system is shutdown, it is possible, to a limited extent, to use this device to control and regulate the operation of the experimental device. Another possible use is the positioning of the supporting arm during the maintenance of the device.

3.3. Algorithms of the System Control. To work with the experimental assembly, it was necessary to create a userfriendly user interface which allows a manual control of the actuator for testing and the definition of the operation during the automatic control of the test equipment. The control system is designed so that the operator can input instructions for the control system using the user interface of the program in an operational workplace (PC). The control system is running on a computing unit NI cRIO 9024. The taskbar of the control system user's interface is shown in Figure 5.

In Figure 6 the main structure of the "control system" is shown, highlighted are the individual parts that constitute the main subprograms of the main algorithm designed for specialized operations: (1) downloading data, (2) initialization of variables, (3) automatic control, (4) position regulation, (5) pressure regulation, (6) control program, and (7) saving the data.

Pressure control is ensured in this subprogram by an algorithm that ensures opening and closing of the electropneumatic valves, based on the comparison of momentary and desired pressures in each PAM (Figure 7), that is, in each executed iteration of the main program. The blocks f1-f4 represent the output control signal for PWM control for each PAM inflation and deflation using the electropneumatic valves.

Position control algorithm (Figure 8) is designed to perform regulatory operations leading to the opening and closing of the electropneumatic valves which are based on data entering the subroutine. Its role in each iteration (repetition) of the control program is to evaluate the state of 


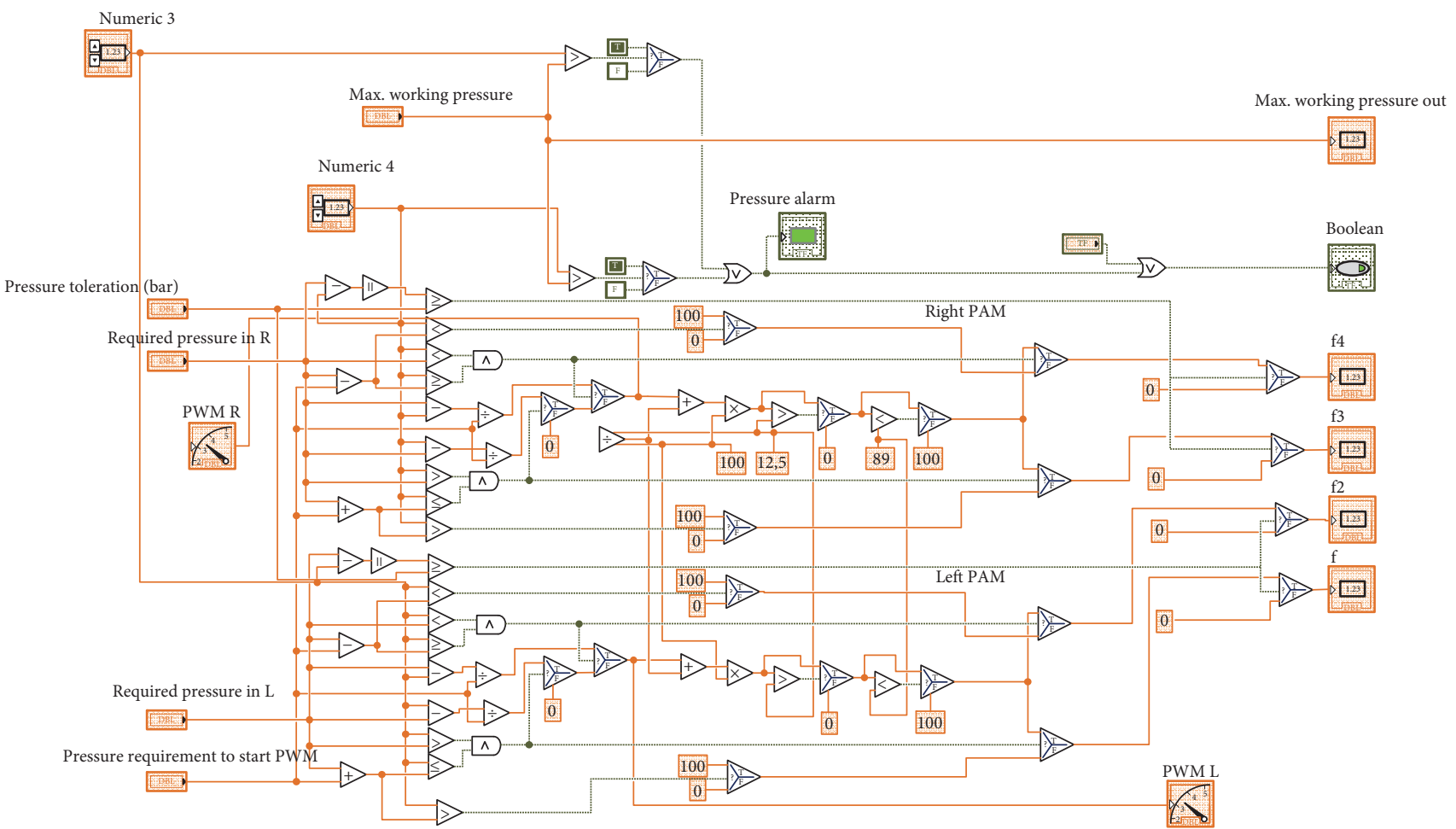

FIGURE 7: Block diagram of the subroutine for the pressure regulation.

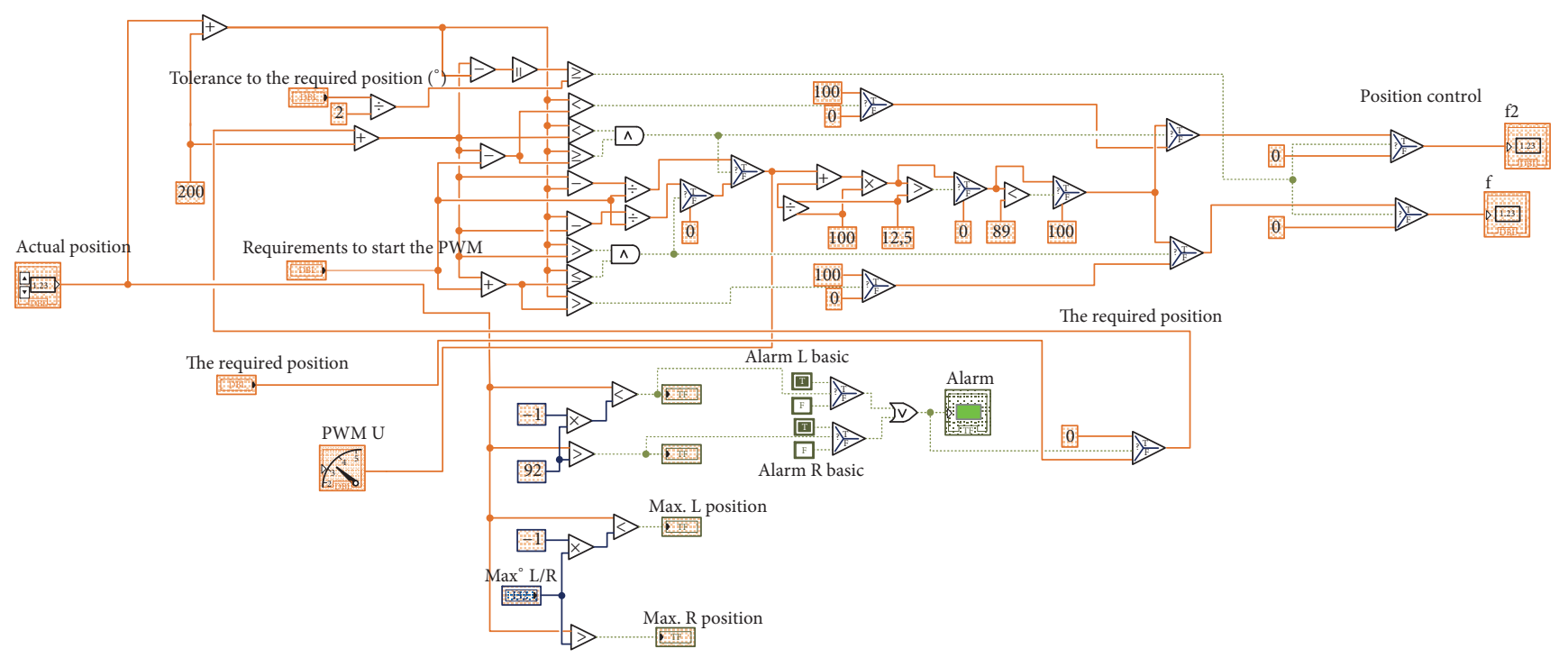

FIGURE 8: Block diagram of the subroutine for the position control.

the controlled system and to decide on its further operation. A subroutine works as a proportional controller.

\section{Experimental Measurements}

4.1. Experimental Measurement Number 1. The experimental actuator was used to carry out experimental measurements for the purpose of obtaining information about the static and dynamic characteristics of the device. The principal aim of the measuring was to describe the limiting conditions of the device operation. The experimental measurement was carried out in a room with a relatively stable temperature. The initial measuring conditions and constant values are presented in Table 2.

Based on the information about the possibilities of the PWM regulation presented in [9], the minimal regulated 
TABLE 2: Initial conditions and constant parameters for experimental measurements.

\begin{tabular}{|c|c|c|c|}
\hline Initial conditions & Value & Units & Annotation \\
\hline Ambient temperature & 24.2 & {$\left[{ }^{\circ} \mathrm{C}\right]$} & - \\
\hline Air tank pressure & Max. 7.5 & [bar] & The pressure at the electropneumatic valves inlet. \\
\hline Pressure sensors power supply & 23 & {$[\mathrm{~V}]$} & Laboratory power supplies. \\
\hline Control valves power supply & 24 & {$[\mathrm{~V}]$} & Laboratory power supplies. \\
\hline Pressure in PAM & 2 & [bar] & Initial operating pressure. \\
\hline Pressure tolerance & 0.05 & [bar] & Setting in GUI of the control system. \\
\hline Position angle of the support arm & 0 & {$\left[{ }^{\circ}\right]$} & - \\
\hline Position tolerance & \pm 1 & {$\left[{ }^{\circ}\right]$} & Setting in GUI of the control system. \\
\hline PWM & $20-100$ & [\%] & $\begin{array}{l}\text { Determined by the control program in each of its cycles } \\
\qquad(f=50 \mathrm{~Hz}) .\end{array}$ \\
\hline Weight & $400 / 25 / 90$ & $\mathrm{~N} / \mathrm{mm} /{ }^{\circ}$ & $\begin{array}{l}\text { Arm without weights (force determined owing to the } \\
\text { shaft axis, with the supporting arm rotation to } 90^{\circ} \text { ). }\end{array}$ \\
\hline
\end{tabular}

TABLE 3: The values of the operational parameters for the experimental measurement number 1.

\begin{tabular}{lcc}
\hline Operational characteristics & Value & Units \\
\hline Positioning (1 series of measurement) & $0,15,30,45,60,75,80,0,-15,-30,-45,-60,-75,-80$ & 5.1 \\
Maximum pressure & 100 & $\left.6{ }^{\circ}\right]$ \\
The number of repetitions of a series & 6 & - \\
Positioning step duration & 16 & {$[\mathrm{bar}]$} \\
Positioning step duration in starting and ending position & 6.5 & {$[\mathrm{~s}]$} \\
Mass of weight & {$[\mathrm{kg}]$} \\
\hline
\end{tabular}

value of the PWM regulation is $20 \%$. The use of lower values would lead to excessive decreases in durability of the electropneumatic valves.

During the experimental operation, the pressure values in each PAM, values of the PWM signals, and rotation and acceleration of the supporting rotational arm were measured. During the measuring, the values listed in Table 3 were applied.

The measuring was carried out under changing ambient conditions with a room temperature in the range of 24.2$26.4^{\circ} \mathrm{C}$ within 100 series of positioning. According to the order given in Table 3, in each series of experimental measurements, the automatic control system was applied to set up the size of the rotation angle of the actuator supporting arm to the desired position. The measurements were performed without setting the limits of the device operation to the recommended operating minima or maxima so that it was possible to observe the behaviour of the antagonistic involvement of the pneumatic artificial muscles mechanism without software restrictions of its operation.

After completing the first experimental measurements, a review measurement of the limit positions of the supporting arm was conducted which were resulting from the restrictions of the operational maximum and minimum pressure in each PAM. Based on the analysis of the measured values, experimental measurement number 3 was performed, whose principle consisted in the ambition to prove the differences in the experimental actuator operation at the start of its operation from the initial state (minimal rotation of the supporting arm) and from the state of the supporting arm maximum rotation.

The data expressing the current position, pressure in each PAM, and the acceleration of the supporting arm obtained based on each series of measurements were presented graphically. Between the last iteration of a series of measurements and the first iteration of the following series of measurements when the system started to respond to the change in position, there was a delay in the range of 0.1-0.4 seconds. Figure 9 shows graphs used for the preliminary analysis of the transient characteristics of the experimental assembly during the operation. To obtain a description of the mechanism behaviour without limiting its operating conditions, its positioning was not restricted through setting up the minimum and maximum operating pressure during this measurement. For the reason of a better readability of the data, the first graph shows the measured data obtained only from the ten consecutive series of measurements.

The processed measured data showed that the measured characteristics recorded in each series of the experiment were not significantly different. In six cases of 100 repeats of the measurement series, there were recorded deviations from the standard course of the measured dynamic characteristics. A presumed cause is a combination of two factors: the operation in the limit positions of the end member of the actuator $\left(75-84^{\circ}\right)$ and the lack of a pressure medium in the air tank. In order to eliminate these phenomena, it was necessary to increase the minimum pressure in the air tank. Another 

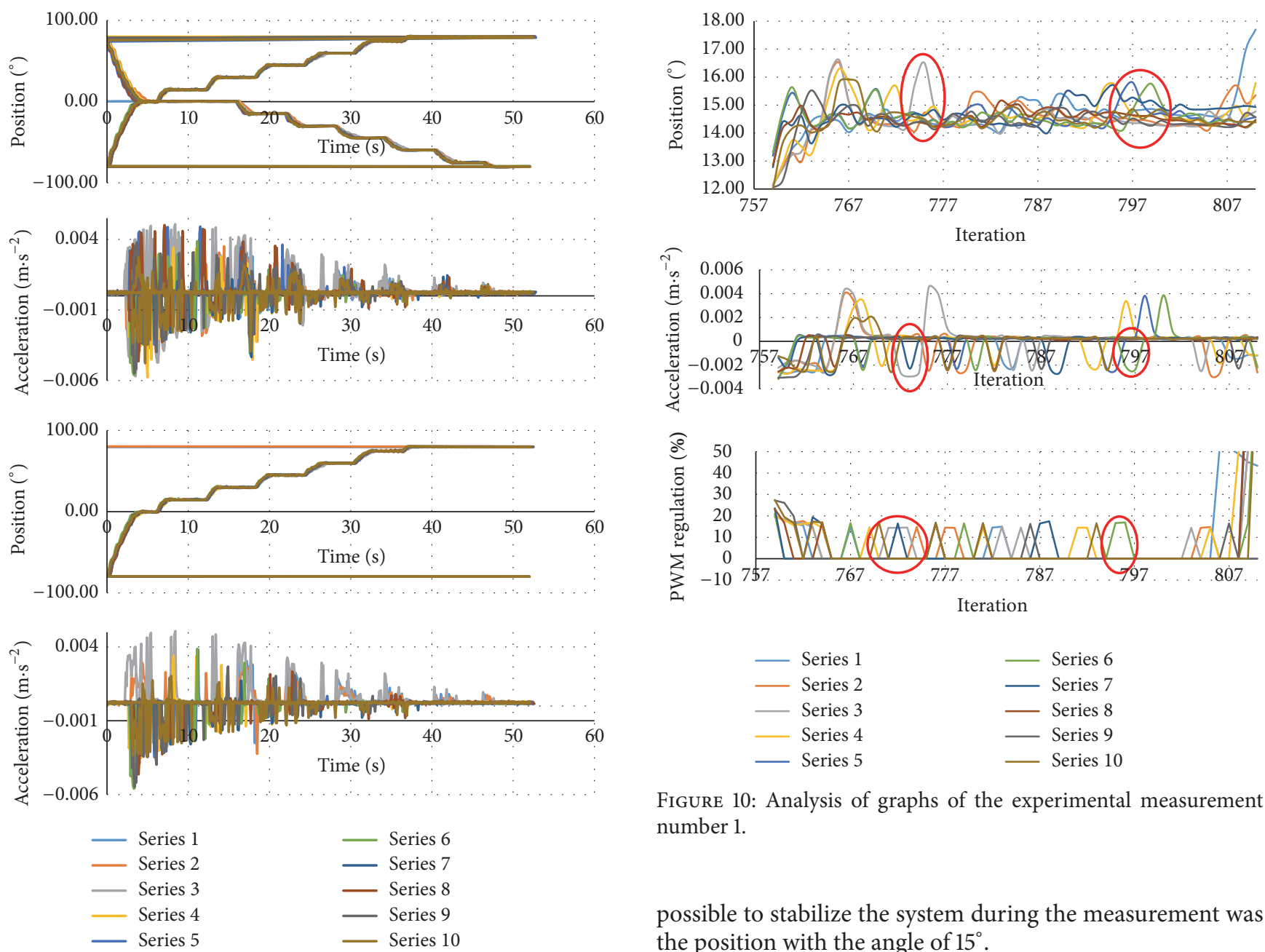

Figure 9: The graphical representation of a current position and the supporting arm acceleration.

reason why these cases occurred was the mechanism operation without limitation of the PAM minimum and maximum operating pressure.

The second graph in Figure 9 shows the support arm acceleration. The acceleration indicates a drop in the size of hydraulic shocks with the increased rigidity of the PAMs mechanisms. For a more detailed analysis, the next graph (the angle of the arm rotation) shows only the data measured during the positioning of the supporting arm in the range $0-84^{\circ}$. Negative values of the acceleration represent the movement of the supporting arm in the positive direction. In case that the supporting arm exceeds the positioning accuracy and the actual position is larger than the required position, the system responds with a feedback in the form of a backward movement. This fact corresponds with positive values of the acceleration.

The rigidity of the system was low at low values of the supporting arm angle. In some cases the device failed to reach a stable state of the desired position during the time interval of one step of the positioning. The lowest value at which it was

FIgURE 10: Analysis of graphs of the experimental measurement number 1 .

possible to stabilize the system during the measurement was the position with the angle of $15^{\circ}$.

Figure 10 shows graphs of the device dynamic characteristics measured while maintaining the position of the supporting arm at $15^{\circ}$. The data are shown in ten series of the measuring. The first graph shows the course of the supporting arm positioning. The principle aim was to maintain the supporting arm in the position with the desired accuracy during its positioning. The definition of the position accuracy caused the arm motion to stop immediately after reaching the accuracy interval. It resulted in reaching a real middle position around $14.65^{\circ}$. Therefore it was necessary to consider the adjustments of the subprogram for the position control. Significant fluctuations in the position are highlighted in the graph. These fluctuations occur predominantly in the positive direction (in the direction of the position increase).

The second graph shows the behaviour of the arm acceleration. The largest fluctuations in the arm position correspond to the largest fluctuations in the arm acceleration. In the series number 3 and number 6 , the position and acceleration waveforms are marked. When compared with the last graph in Figure 10, there can be seen a direct relation between the occurrence of hydraulic shocks and opening (closing) of electropneumatic valves or between the formation of hydraulic shocks, the size of the PWM regulation, and time of the PWM operation.

Figure 11 shows the waveforms of the measured variables in the fifth series of the experiment. Based on a comparison of 

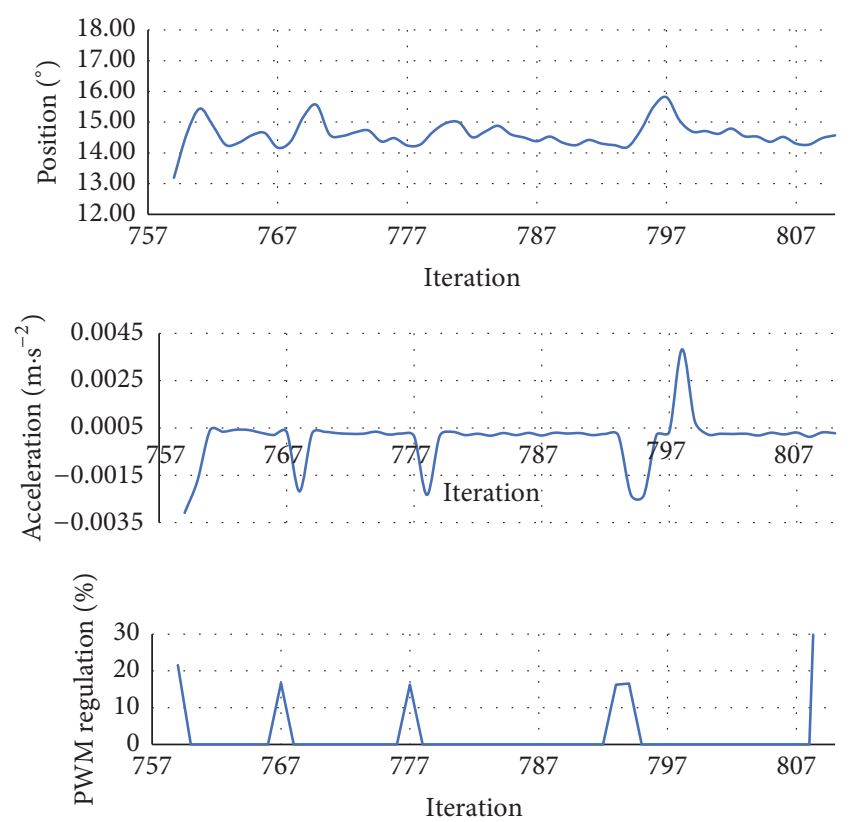

- Series 5

Figure 11: Analysis of the graphs of the series number 5 of the experimental measurement number 1.

the graph of the acceleration and size of the PWM regulation, a dependence of these phenomena is noticeable. The assembly of the experimental manufacturing device responds to the electropneumatic valve opening by the arm movement which causes a dynamic shock (change in speed or acceleration) in the direction of the desired movement. Closing of the electropneumatic valve gives rise to the effect of inertia that acts upon the supporting arm causing a pose overshoot in the opposite direction. There is evident time difference between the opening and closing of the valve and the system response in the range of one control program iteration. One iteration is considered the minimum time unit (experimental measurement number 1). While the experimental actuator was moving into different positions, there were recorded the same actions as mentioned above. A supporting arm angle of $15^{\circ}$ was used to represent these actions because there is low rigidity of the whole system and these actions are better viewable.

Based on the data processing obtained in the experimental measurement number 1 , the following conclusions have been taken:

(i) For the implementation of relevant measurements, it is sufficient to perform only ten repeats of a properly performed experiment.

(ii) Identified dynamic shocks generated in experimental involvement are directly linked to the compressed medium supply system of PAMs.

(iii) The algorithm of a position control should be reconsidered and adapted to new requirements with regard to the positioning accuracy; subsequently, changes have been made in the control algorithm.
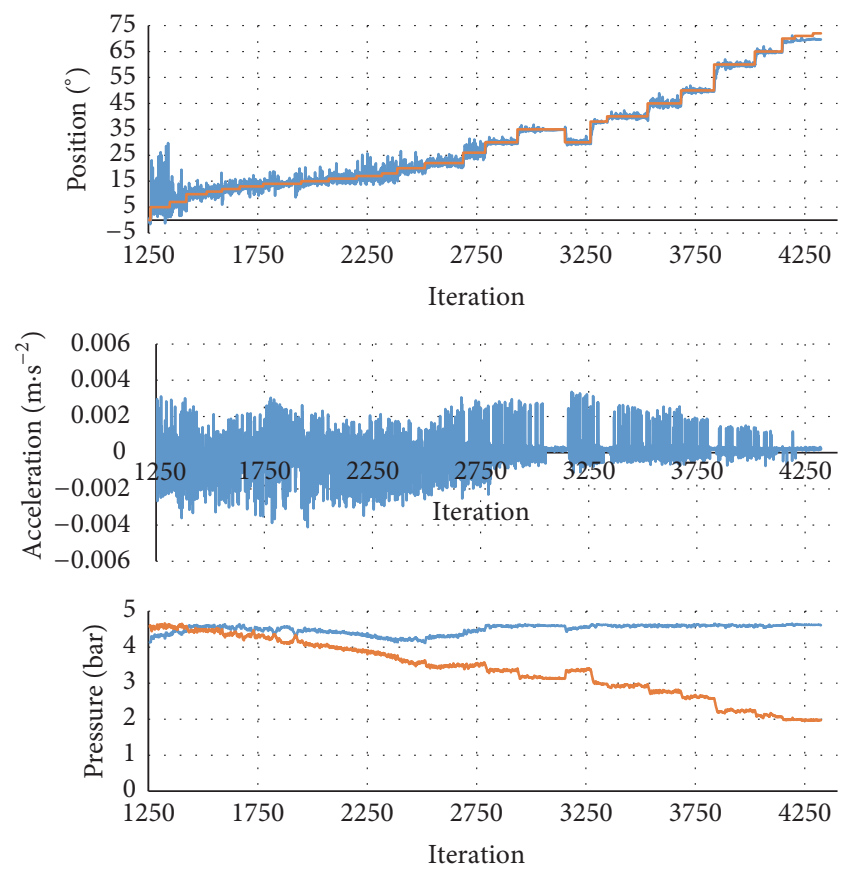

FIGURE 12: A graphical representation of the experimental measurement number 2; positioning accuracy $=1^{\circ}$.

(iv) Taking into account the impact of rigidity of the PAMs on the formation of dynamic shocks in the pneumatic system as well as the impact on the positioning repeatability in a full operational interval, it is possible to determine the interval of the operating positions in which, according to preestablished requirements, it will be possible to achieve an adequate positioning accuracy and sufficient rigidity of the system to ensure steady required static and transient characteristics of the mechanism.

4.2. Experimental Measurement Number 2. Based on the results of the first experimental measurement, changes to the control system were performed. The experimental measurement number 2 was performed to verify the assumptions related to the system behaviour when minimum and maximum operating pressures were set. The experiment was performed by a gradual enlargement of the supporting arm deviation of small values. The aim was to achieve the most accurate description of the mechanism rigidity impact on the actuators ability to stabilize itself in time. The measurement started after the stabilization of the arm in the position $0^{\circ}$. Deviations in the position started to be slowly stabilized in the required accuracy (the positioning accuracy selected to $\pm 1^{\circ}$ ) in the position range of $34^{\circ}$ and higher (Figure 12). In the range of the positions $0-34^{\circ}$, a supporting arm oscillated around the requested position, but it was not possible to reach its stability, or its stabilization took a long time. It was due to the use of the PAMs control system which worked too fast. In case of an excessive oscillations of the arm, the size of the arm rotation was recorded by the control system. The control system has an algorithm applied in such situations, where an 

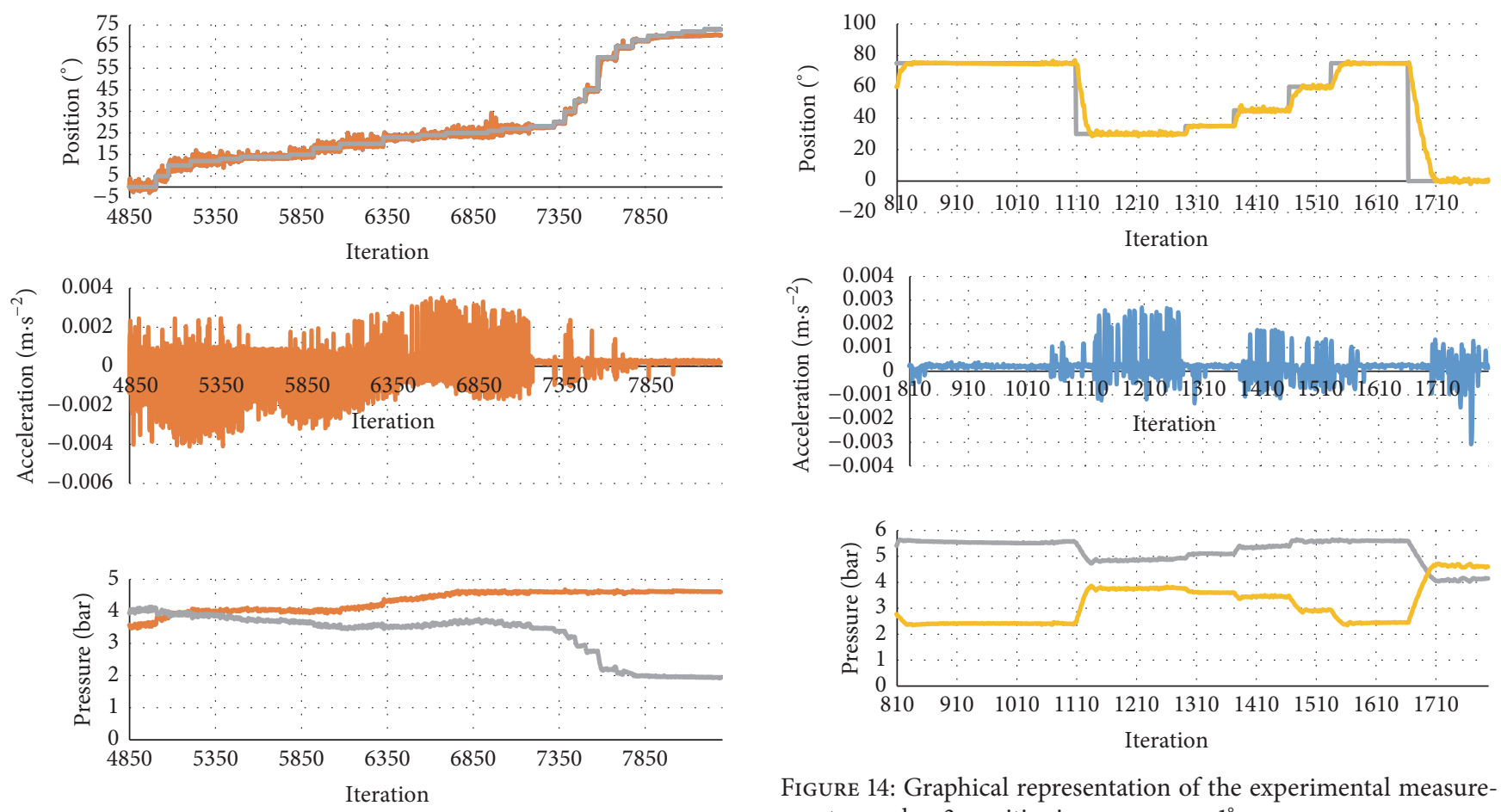

FIGURE 13: Graphical representation of the experimental measurement number 2 ; positioning accuracy $=1.5^{\circ}$.

action signal is launched to regulate the position of the arm in the opposite direction.

When using limits of minimum and maximum operating pressures in each PAM, the position of max. $70.00^{\circ}$ can be reached. This measurement confirms the validity of the supplemented functions of the algorithm that do not allow exceeding the set operating pressures in the PAMs.

Figure 13 shows waveforms of the position, acceleration, and pressure when positioning with the accuracy of $1.5^{\circ}$. In the graphs a considerably more stable operation with lower rates of vibration can be seen after exceeding the position of $25^{\circ}$. Pressure characteristics in each muscle differ from previous measurements. This is due to the position control by the method when one of the muscles is not used as a pneumatic spring with a constant pressure. In order to eliminate the pressure differences, before starting measurement in each series of the experiment, it is useful to perform the so-called initialization series to set up the device.

\subsection{Experimental Measurement Number 3: Measurement after} the Initialization Series. Experimental measurement number 3 was carried out after the initialization series (a device setting up) had been performed. The arm was positioned to the maximal achievable position when the pressure was set to $5.5 \mathrm{bar}$ (in the control program, a maximum operating pressure predetermined to 6 bar with a safety fuse of 0,5 bar). Positioning was done with the accuracy of $\pm 1^{\circ}$. Graphical representation of the experimental measurements number 3 is shown in Figure 14. Stability of the mechanism was achieved at the position angle of $30^{\circ}$. This allows improving the operation stability.

Due to the improvement of the mechanism stabilization in a specific position, it is also possible to extend the range of positions reaching a stable operation by the value of $4^{\circ}$.

\section{Evaluation of the Results of Experiments and Proposed Modifications of the Experimental Device}

The aim of the experiments was to describe transient phenomena occurring inside the PAMs. The results have confirmed the hypothesis about the PAMs and the air tank and time of its effect. At the same time there was confirmed the possibility of automation of the system using appropriate control algorithms for achieving theoretically possible positioning accuracy without the need for the implementation of complicated hardware nodes.

From the point of view of the positioning accuracy with major differences between positions, hydraulic impacts were not so important compared to minor changes in the position. A pressure difference has a significant impact on the speed of the supporting arm. Each PAM is controlled by an electropneumatic valve with the PWM. A high pressure difference significantly affects the arm movement acceleration or the amount of kinetic energy that is transferred to the supporting arm in a time period during which the valve is open. The arm positioning accuracy also depends on the way of stopping the supporting arm, or it depends on the method of a braked movement of the supporting arm [21-23].

Based on the nature of potential functionalities of the mentioned pneumatic actuator, it follows that the system braking is performed only by a sudden stop or by the PWM. 
With a sudden stop of the supporting arm, under the effect of inertial force, the misalignments of the supporting arm occur. Taking into account the nonlinearity of the entire mechanical system and the subsequent oscillation of the arm around the equilibrium position (as seen from the experimental measurements, the equilibrium position need not be equal to the position required), the size of this vibration is directly dependent on the supporting arm acceleration.

In case of a high pressure difference, there is an adequate response of the pneumatic system in the form of a hydraulic shock. This results in the deviation of the supporting arm position from its equilibrium position. The size of the deviation corresponds to the size of the differential pressure. Experimental measurements have shown that at a low rigidity of the mechanical system the deviations of the position are above the range of the required positioning accuracy specified to $1^{\circ}$, max. $1 \cdot 5^{\circ}$. After exceeding the positioning accuracy, the control system automatically ensures the arm to move in the opposite direction. To adjust the position of the arm, a certain period of time is required due to which an undesirable delay occurs. Such a delay may require a lower speed of handling and manufacturing operations in flexible long-term operations. In some cases this can be recognized as a bottleneck in the production system. In such cases, the use of such a system would not be possible. The use of such a system is not suitable in fluid material handling.

Operating range of positions in which such a system allows its stable operation is greatly influenced by a pressure difference between the source of the working medium and the pressure in the pneumatic artificial muscles, rigidity of each PAM, and a load on the supporting arm (torque acting on the shaft).

Rotating of the actuator arm is provided by using four electropneumatic valves enabling the proportional regulation (PWM). The applied PWM regulation allows reducing the amount of the inflated and deflated fluid from the fluid system in time. The PWM regulation is not possible in its entirety. In practice, it is verified that with this system it is necessary to limit the use of the PWM control to the range of 20$100 \%$. This restriction is required to achieve an adequate durability of electropneumatic valves. In the control system, it is limited to the interval of $20-89 \%$. In case that the value of the needed PWM in a given iteration is greater than $89 \%$, the system automatically uses the value of $100 \%$. The PWM regulation causes a gradual slowdown of the supporting arm movement. However, if the formation of hydraulic shocks is taken into account that accurately correspond to the opening and closing of the electropneumatic valves, it is obvious that the use of a time smoother regulation would be preferable. A better solution might be a regulation of a gradual closing of the valve with a continuous flow of the working fluid, or a regulation that would prevent from hydraulic shocks formation in a pneumatic system of the PAMs mechanism.

A control utilizing the PWM regulation has improved properties compared to a control using a maximum pressure difference dependent on the current pressure in the air tank. On the one hand, a limitation of the PWM operating range to only $20 \%$ and higher causes a slight slowdown of the arm movement. On the other hand, there is the impact of hydraulic shocks arising even with the lowest PWM application. With a lower rigidity of a mechanism, under the influence of hydraulic shocks it is impossible to stop the supporting arm in the narrowest range around the desired position.

Despite many disadvantages described above, the regulation of the supporting arm positioning to the desired position using the PWM allows obtaining a relatively stable and repeatable positioning in a significantly greater range of a rotational angle of the supporting arm.

For the experimental actuator, designs of alternative involvements of technical equipment of the actuator have been created. The proposed alternatives of changes of the experimental actuator differ from the original involvement diagram. The main differences are in elements allowing a continuous pressure change [24-26].

\section{Conclusion}

The main objective of the described measurements was to elaborate the methodology of online monitoring and control of propulsion systems in manufacturing technology, specifically for manufacturing devices with artificial muscles in order to achieve optimal results of the positioning accuracy.

The paper consists of two parts. The first part of the paper contains a description of the experimental device with a drive based on pneumatic artificial muscles (using the PWM), identification of its physical possibilities, and a prediction of a complete mechanism behaviour. The second part describes the realization of experimental measurements, analysis and assessment of the measurements results, and a design of the control system modification or a modification of the entire actuator. The article deals with the evaluation of the state and the possibilities of the experimental involvement. In order to achieve the ability to automatically control the position of the supporting arm, a proposal for the actuator optimization has also been described.

The device was used to carry out experimental measurements. Their purpose was to analyze the behaviour of the propulsion system based on a pair of the PAM assembled in antagonistic involvement under different operation conditions. The experiments were focused on the examination of the positioning accuracy. The data about the current pressure inside each PAM, a value of the PWM, and a current position and acceleration of the supporting arm have been recorded. Based on the processed and evaluated measured data, the characteristics of the actuator and processes taking place inside its components during the operation have been described. Based on the graphic representation of the measured variables, it was possible to identify the source of excessive fluctuations in the position of the supporting arm as well as the nature of the hydraulic shock occurrence in the experimental pneumatic actuator system. The measured data have been statistically analyzed. Results of the data processing showed the effect of the PAMs rigidity and the size of the arm rotation angle on the stability of the device operation and the accuracy of the arm positioning.

As follows from the carried out experimental measurements, it is possible to automate the systems with the PAMs by appropriate algorithms and using the appropriate hardware 
equipment to achieve the required positioning accuracy in the technically useful range. The measurements showed that the PAMs systems (described in this article) are able to operate with the absolute positioning accuracy $1^{\circ}$ and less in compliance with the range of oscillation and the value of the gravitational overload. It is also possible to interpret the given findings that by appropriate algorithmisation and determination of time increment of the measurements in combination with the change in the rigidity of the system and its inversion it is possible to achieve a high accuracy even in a narrow range of the change in the position in relation to a baseline.

After the evaluation of the course and results of the first experimental measurement, there have been modifications of the software base of the control system performed by adding the limits for a maximum and minimum operating pressure in each PAM.

The actuator has been provided with acceleration sensors to identify the shocks inside the pneumatic artificial muscles, or the sensors of the changes in the torque acting on the actuator arm.

Based on the data obtained by the experimental measurements, several alternatives have been proposed to supply the elements to the experimental device that would allow a continuous deceleration, or the acceleration of the supporting arm movement, eventually the reduction of the impact of the pressure difference value between the air tank and the PAMs (to stop the formation of hydraulic shocks with the undesirable values).

Within the solution of these issues, attention has been preferably paid to practical options to control a particular experimental device. Searching for other alternatives to describe the processes running inside the mechanism after the device enlargement by adding the proposed modifications in order to reduce the impact of the hydraulic shocks on the device operation could also be interesting.

\section{Competing Interests}

The authors declare that there is no conflict of interests regarding the publication of this paper.

\section{Acknowledgments}

This paper is supported by the project VEGA 1/0338/15 "Research of effective combinations of energy sources on the basis of renewable energies." This paper is supported by the Slovak Research and Development Agency under the Contract no. APVV-15-0602.

\section{References}

[1] Kopečný and F. Šolc, "McKibben pneumatic muscle actuator in robotics," AT\&P Journal, vol. 2, p. 62, 2003.

[2] L. Kárník, R. Knoflícek, and J. N. Marcincin, Mobilní Roboty, Márfy Slezsko, Opava, Czech Republic, 2000.

[3] W. Huang, Shape memory alloys and their application to actuators for deployable structures [Ph.D. thesis], University of Cambridge, 1998.
[4] K. C. Wickramatunge and T. Leephakpreeda, "Study on mechanical behaviors of pneumatic artificial muscle," International Journal of Engineering Science, vol. 48, no. 2, pp. 188-198, 2010.

[5] I. Díaz, J. J. Gil, and E. Sánchez, "Lower-limb robotic rehabilitation: literature review and challenges," Journal of Robotics, vol. 2011, Article ID 759764, 11 pages, 2011.

[6] Z. Šitum, "Pneumatski mišić kao aktuator," Znanstvenopopularni časopis Sustavi, vol. 3, no. 5, pp. 54-60, 2009.

[7] M. Rimár, P. Šmeringai, M. Fedák, and Š. Kuna, "Alternatives for real time positioning control system in mechatronic system with pneumatic artificial muscles," International Journal of Engineering Research in Africa, vol. 18, pp. 3-11, 2015.

[8] J. Pitel', M. Balara, A. Hošovský, and M. Tóthová, Pneumatické Umelé Svaly: Modelovanie, Simulácia, Riadenie, Technická Univerzita v Košiciach, Košice, Slovakia, 2015.

[9] Š. Kuna, Výskum metód real time monitoringu a diagnostiky výrobných strojov [dissertation work], Technická Univerzita $\mathrm{v}$ Košiciach, Košice, Slovakia, 2014.

[10] F. Zezulka, P. Fiedler, and Z. Bradáč, Prostředky Průmyslové Automatizace, Vysoké Učení Technické v Brně, Fakulta elektrotechniky a Komunikačních Technológií, Brno, Czech Republic, 2002, http://www.spsei.cz/att/soubory/prostredky_ prumyslove_automatizace.pdf.

[11] R. Ramasamy, M. Juhari, M. Mamat, S. Yaacob, N. Mohd Nasir, and M. Sugisaka, "An application of finite element modelling to pneumatic artificial muscle," American Journal of Applied Sciences, vol. 2, no. 11, pp. 1504-1508, 2005.

[12] H. P. Anh and N. T. Nam, "Modeling and adaptive selftuning MVC control of PAM manipulator using online observer optimized with modified genetic algorithm," Engineering, vol. 3, no. 2, pp. 130-143, 2011.

[13] G. K. Klute, J. M. Czerniecki, and B. Hannaford, "McKibben artificial muscles: pneumatic actuators with biomechanical intelligence," in Proceedings of the IEEE/ASME International Conference on Advanced Intelligent Mechatronics (AIM '99), pp. 221-226, Atlanta, Ga, USA, September 1999.

[14] D. Plettenburg, "Pneumatic actuators: a comparison of energyto-mass ratio's," in Proceedings of the 9th International Conference on Rehabilitation Robotics (ICORR '05), pp. 545-549, IEEE, Chicago, Ill, USA, June 2005.

[15] “FESTO: Fluidní svaly DMSP/MAS”, 2004, https://www.festo .com/cat/en-gb_gb/data/doc_SK/PDF/SK/MAS_SK.PDF.

[16] T. Szepe, "Accurate force function approximation for pneumatic artificial muscles," in Proceedings of the 3rd IEEE International Symposium on Logistics and Industrial Informatics (LINDI '11), Budapest, Hungary, August 2011.

[17] J. L. Serres, Dynamic characterization of a pneumatic muscle actuator and its application to a resistive training device [dissertation work], Wright State University, Dayton, Ohio, USA, 2008.

[18] J. Pitel' and M. Balara, "Pneumatický umelý sval-perspektívny prvok mechatroniky," AT\&P Journal, vol. 4, no. 1, pp. 59-62, 2009.

[19] P. Šmeringai, Výskum metód online monitorovania výrobných zariadení s umelými svalmi [Ph.D. dissertation], Technical University in Košice, 2016.

[20] V. T. Jouppila, S. A. Gadsden, G. M. Bone, A. U. Ellman, and S. R. Habibi, "Sliding mode control of a pneumatic muscle actuator system with a PWM strategy," International Journal of Fluid Power, vol. 15, no. 1, pp. 19-31, 2014. 
[21] D. W. Repperger, C. A. Phillips, A. Neidhard-Doll, D. B. Reynolds, and J. Berlin, "Actuator design using biomimicry methods and a pneumatic muscle system," Control Engineering Practice, vol. 14, no. 9, pp. 999-1009, 2006.

[22] A. Šmeringaiová, I. Vojtko, and K. Monková, "Experimentelle Analyse der Dynamik von Zahnradgetrieben-Teil 1," TMTechnisches Messen, vol. 82, no. 2, pp. 57-64, 2015.

[23] A. Šmeringaiová, I. Vojtko, and K. Monková, "Experimentelle Analyse der Dynamik von Zahnradgetrieben-Teil 2," TMTechnisches Messen, vol. 82, no. 4, pp. 224-232, 2015.

[24] A. Panda, M. Prislupčák, J. Jurko, T. Krenický, and M. Jančík, "Evaluation of vibration parameters under machining," Key Engineering Materials, vol. 669, pp. 228-234, 2016.

[25] Š. Salokyová and T. Krenický, "Analysis of the effects of factors in relation to vibration of technological head during the splitting of construction steels through hydro-abrasive splitting," Key Engineering Materials, vol. 669, pp. 212-219, 2016.

[26] J. Zajac, D. Mital, P. Michalik, and D. Mital', "Verification of process fluids in mass production," in Proceedings of the 7th International Congress of Precision Machining, Key Engineering Materials (ICPM '13), vol. 581, pp. 554-559, Miskolc, Hungary, October 2013. 

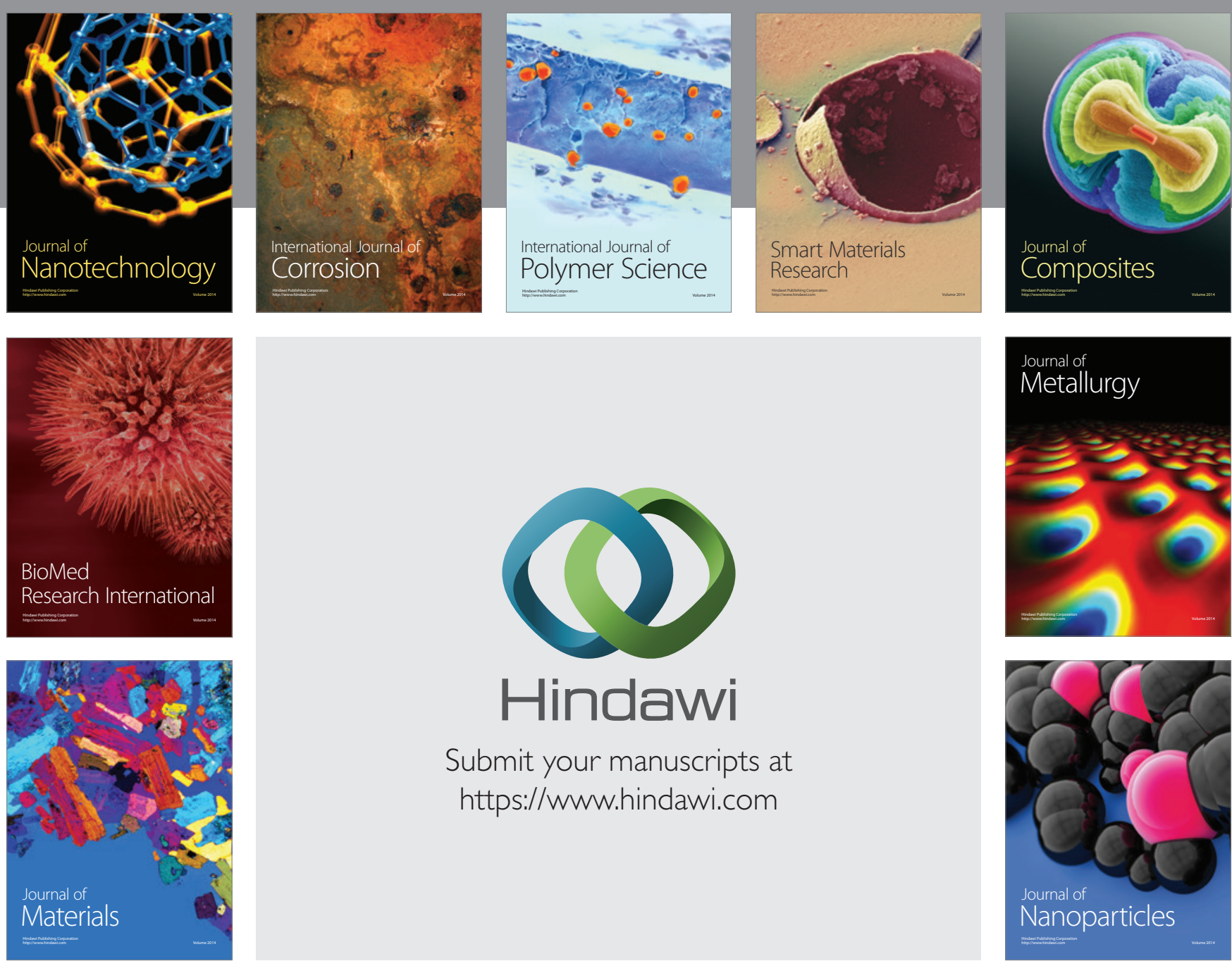

\section{Hindawi}

Submit your manuscripts at

https://www.hindawi.com

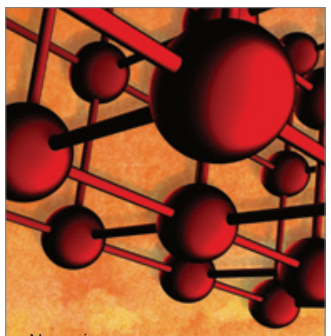

Materials Science and Engineering
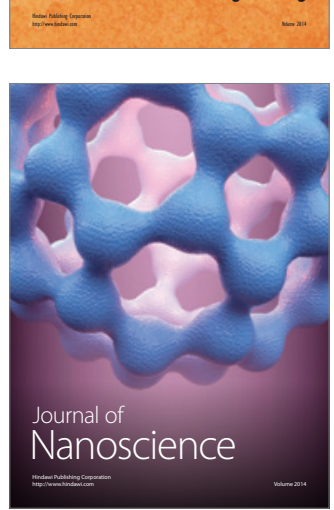
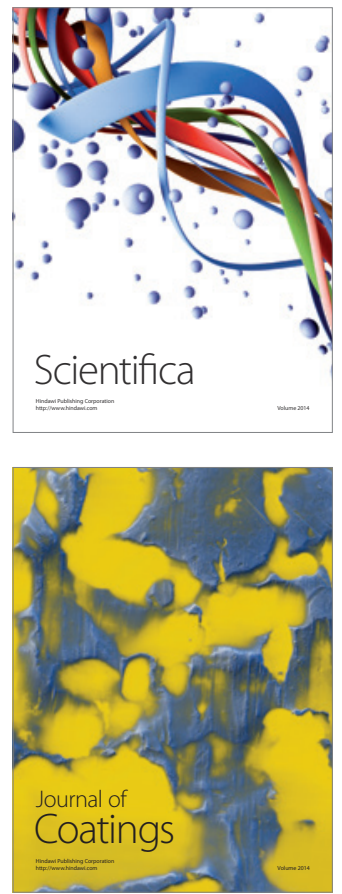
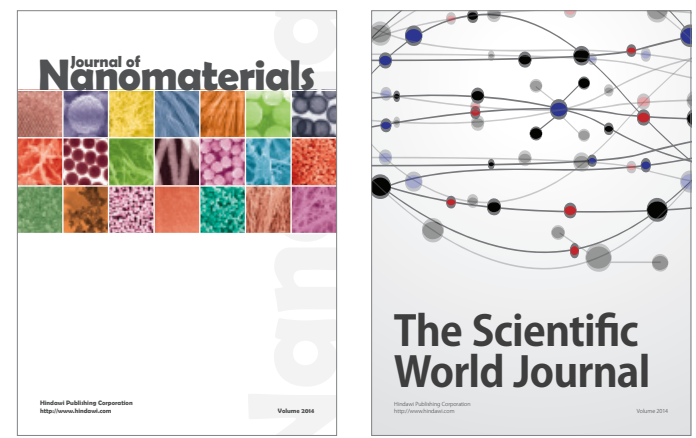

The Scientific World Journal
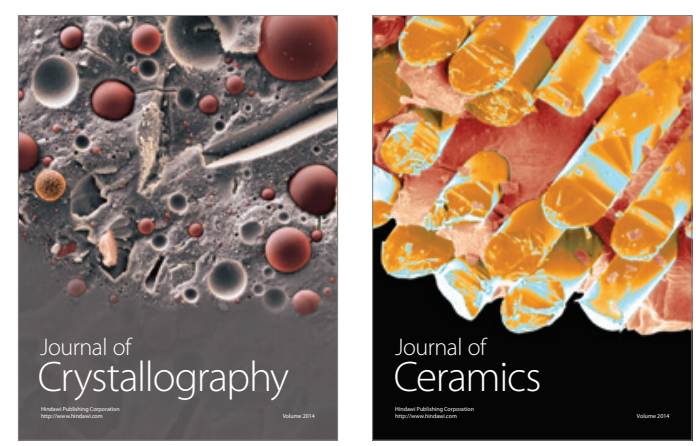
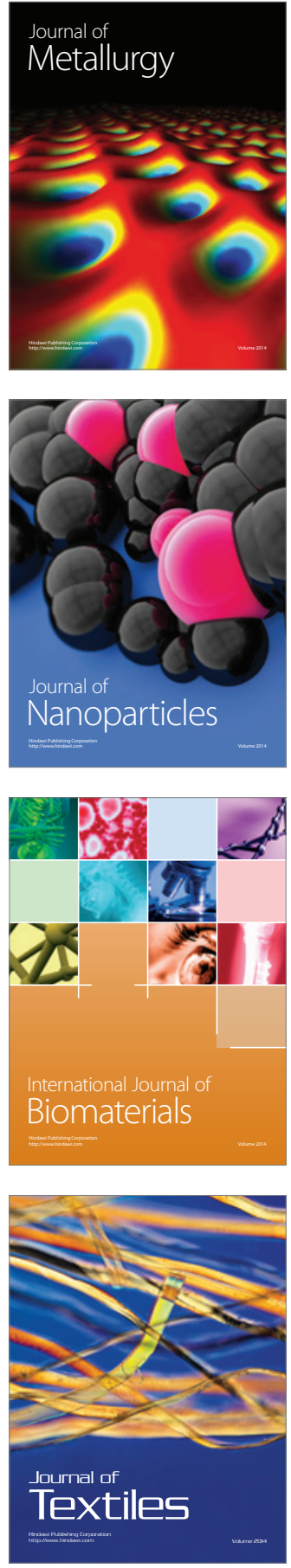